\title{
A Study of Ultrasound and CT Guided Therapeutic Drainage of Intra-Abdominal Collections and Abscesses
}

\author{
Senthil Anbumani ${ }^{1}$, Anita Soundarapandian ${ }^{2}$ \\ ${ }^{1}$ Associate Professor, Department of Radiology, ACS Medical College and Hospital, ${ }^{2}$ Associate Professor, Department of \\ Radiology, Saveetha Medical College and Hospital, Chennai, India \\ Corresponding author: Anita Soundarapandian, Flat 3A, Navanya Apartments, 3/31, 12th Street, B Block, Annanagar East, \\ Chennai - 600102, India
}

DOI: 10.21276/ijcmsr.2018.3.3.28

How to cite this article: Senthil Anbumani, Anita Soundarapandian. A Study of ultrasound and CT guided therapeutic drainage of intra-abdominal collections and abscesses. International Journal of Contemporary Medicine Surgery and Radiology. 2018;3(3):C126-C130.

\section{A B S T R A C T}

Introduction: Intra-abdominal abscess remains a significant cause of morbidity and mortality in the patient population. Though there have been significant advances in treating them, it is still difficult to diagnose and treat. In fact it is the most dreaded complication of surgery. The aim of the study was to demonstrate the safety and efficacy of percutaneous drainages of intra-abdominal abscesses and collections when done under image guidance. Another aim was to demonstrate that this can be done even in ill patients who are not fit for general anesthesia.

Material and methods: CT guided drainage of abscesses and collections were performed for 102 patients over a period of one year. Amongst the patients, there were 88 men and 14 women. The ages ranged from 16 to 72 . A 128 slice CT scanner (Philips) was used for guidance.

Results: Of the 102 drainage, 70 were done under CT guidance and 32 under US guidance. The abscesses were completely drained percutaneously in 91 patients (89.2\%), hence avoiding invasive surgery.

Conclusion: image guided catheter drainage of an intra-abdominal infective collection is a safer alternative to open surgical drainage both in healthy patients, and even more important in patients who are not fit for general anesthesia. CT guidance proved to be a better modality both in complete drainage and in avoiding complications.

Key words: Abscess, Catheter Drainage, Pigtail, Percutaneous, CT Guidance, Ultrasound Guidance

\section{INTRODUCTION}

Infective collections in the abdomen can cause significant morbidities and sometimes mortalities. Even though there have been numerous advances, these still remains a difficulty to both diagnose as well as treat. It is one of the most feared complications of surgery. ${ }^{1}$ Most abscesses will end up requiring drainages. Open surgical drainage with adjuvant antibiotics used to be the traditional treatment protocol. ${ }^{2}$ Recently however, sophisticated percutaneous drainage methods are evolving as an important therapeutic tool in the management of this problem. ${ }^{3}$ There have been tremendous developments in the form of advanced CT scanners, novel catheters and other equipments. Consequently, image guided therapeutic drainage have now gained widespread usage in intra abdominal abscesses.

It was in 1953, that percutaneous drainage along with adjuvant antibiotics was used for the very first time to effectively drain a liver abscess. From that time onwards percutaneous abscess drainage has been in use and constantly modified, and updated to drain collections in various parts of the body. ${ }^{4}$ Image guidance for drainage came into force initially when Gronvall et al performed of four cases drained under ultrasound guidance. Later, Gerzof et al set a successful protocol for percutaneous abscess drainage using both ultrasound and computed tomographic guidance in twenty nine cases, all of which were successfully drained. Later, it was Haaga and Weinstein who showed that the safety and effectiveness of percutaneous abscess drainage procedure was better with CT guidance, which they demonstrated in thirty three patients. ${ }^{5}$

The aim of the study was to demonstrate the safety and efficacy of percutaneous drainages of intra - abdominal abscesses and collections when done under image guidance. Another aim was to demonstrate that this can be done even in ill patients who are not fit for general anesthesia.

\section{MATERIAL AND METHODS}

This was a prospective interventional case study done in Saveetha Medical College between August 2015 to September 2016, after obtaining the approval of the institutional ethics committee.

CT guided drainage of abscesses and collections were done for 102 patients over a period of one year. Amongst the patients, there were 88 men and 14 women. The ages ranged from 16 to 72 .

An 128 slice Philips CT scanner or a 16 slice Siemens CT scanner were used for CT guidance. Ultrasound guidance 
was by a Philips ultrasound machine.

Informed consent was obtained from all the patients prior to the procedure after counseling them about the technique, after care and possible complications.

Drainage catheters used in our study was hydrophilic, multipurpose pigtail drainage catheters with locking mechanism; sizes 8 to $14 \mathrm{~F}$.

Dilators were Standard and Coons dilator series.

Initial aspiration needle used was a 20 to $22 \mathrm{G}$ Chiba needle. Guidewires used were 0.035 or 0.038 stainless steel or nitinol, standard stiffness, Amplatz guidewire.

\section{Inclusion criteria}

Solitary, defined, safely accessible collections and abscesses were included in the study.

Sick patients, post operative patients and patients who were deemed unfit for general anesthesia were also included in the study. ${ }^{6,7}$

\section{Exclusion criteria}

Illdefined phlegmons were excluded from the study, since it may lead to worsening of the infection.

Multiple abscesses were excluded from the study.

Multiple loculations within the abscess were considered as relative contra indications, since it may require multiple accesses and therefore multiple catheters.

Hydatid cysts are absolute contra indications due to the very high risk of anaphylaxis.

Percutaneous route is not the access of choice for pelvic abscesses, which require a transvaginal or transrectal route.

Altered prothrombin time and INR is a relative contraindication to percutaneous catheter drainage. ${ }^{8,9}$

\section{Technique}

Patient would be started on pre-procedural antibiotics.

The first step would be to accurately locate the abscess using CT and ultrasound.

Multiplanar CT scored over ultrasound when there were bowel loops / air overlying the abscess because of its inherent advantage over ultrasound in presence of gas, bone and bowel loops overlying the abscess. However ultrasound is essential in draining liver abscesses to assess the surrounding parenchymal vascularity. These patients also required an intravenous contrast administration for the same purpose. In contrast enhanced CT, the abscesses appeared as thick irregular walled lesions, which showed a central non enhancing liquefied area. ${ }^{11}$

The next step was a diagnostic aspiration using a wide bore, long stemmed needle. CT guidance was the preferred modality, except in large superficial abscesses and liver abscesses where ultrasound guidance with color Doppler was preferred. ${ }^{12}$

The needle was selected based on the distance of the center of the abscess from the surface; this was measured either in ultrasound or CT and the access point was marked.

The needle gauge was selected as 18 to $20 \mathrm{G}$ depending on how thick the collection appeared on imaging.

Under strict aseptic precautions, the local area was prepared. Local anesthetic was injected. With image guidance, the needle with negative suction was inserted and advanced to the point already intended. Around 10 to $20 \mathrm{ml}$ of pus / fluid was aspirated for lab work up.
Intravenous broad spectrum antibiotics are mandatory before actual drainage catheter insertion to protect against possible sepsis when the abscess wall is traversed. ${ }^{13,14}$

Planning the approach for drainage needs careful observation and planning, and is easily the most important step in the procedure. The steps to be considered are the point of cutaneous entry, angle of entry and depth of entry. Intervening organs and vessels need to be avoided to prevent organ damage and internal hemorrhage. The aim would be to access the most dependant part of the collection, since this would aid in complete drainage.

Catheter access route is best delineated by using CT. ${ }^{15}$

The access was by a modified Seldinger's technique. Under CT and / or ultrasound guidance, the catheter was inserted. Sometimes fluoroscopic guidance was additionally employed. A guidewire with a "J floppy tip" is then inserted through the access needle, and advanced till it reached and coiled against the dependant wall of the abscess. The needle is removed and angiographic dilators of caliber 8 to $12 \mathrm{~F}$ were passed over the inserted guidewire. Once adequately dilated, the catheter was inserted over the guidewire, and was advanced with image guidance until it coiled along the dependant wall of the abscess cavity. The caliber of the catheter is usually between 9 to $12 \mathrm{~F}$ and is usually a pigtail catheter. Pigtail catheters contain multiple side holes, and preferably should be so placed so that all the side holes are within the abscess cavity.

Trochar technique: when the abscess is large and superficial with a safe direct access route, a one step trochar technique is used. After confirming the position of the catheter within the abscess cavity, the trochar is removed, and the pigtail is allowed to coil within the cavity.

In our study, only the Seldinger technique was used in abscess drainage, since all abscesses were deep without an unimpeded access.

Once the catheter is seated, all pus should be aspirated. With copious irrigation of normal saline, till the returns are clear, the majority of pus can be initially removed. The catheter was then firmly secured to the skin using sutures and placed in a sealed drainage bag. ${ }^{16,17,18}$ Post procedural monitoring the catheter with regular saline flushing is essential to maintain patency. The hourly volume and the nature of the draining fluid / pus was charted and maintained. ${ }^{19,20}$

Two types of catheters can be used for percutaneous drainage.

i) Pigtail catheters: These multiple side-hole catheters are available in various sizes. In this study 9-12 $\mathrm{F}$ pigtail catheters were used, the larger ones being used for the more viscous collections.

ii) Sump catheters: These are multiple side-hole large bore (14 F) catheters inserted over a long metal introducer. The fitted introducer stylet provides support for the catheter and avoids the frequent problem of tissue resistance from body wall or fibrous tissue; it often obviates the need for dilators. These catheters are introduced using the trocar technique. Sump catheters were not used in our study.

\section{Criteria for catheter withdrawal \\ Clinical criteria}

- Defervescence 
- Decreased WBC Count

- Improved appetite and overall condition.

\section{Radiologic Criteria:}

- Cavity collapse as evidenced by

- CT

- US

- Contrast injection,

- No evidence ofany lobulation/multiple collections.

\section{Catheter Criteria}

- Drainage change from sanguinous / purulent to serous

- Marked decrease in drainage clearing of drainage contents.

All the patients were followed for a period of minimum 3

\begin{tabular}{|l|c|}
\hline Location of abscesses & Number of patients \\
\hline Intrahepatic & 32 \\
\hline Iliopsoas & 24 \\
\hline Splenic & 2 \\
\hline Renal & 4 \\
\hline Subdiaphragmmatic & 26 \\
\hline Paracolic gutter & 6 \\
\hline Subhepatic & 8 \\
\hline Total & 102 \\
\hline $\begin{array}{l}\text { Table-1: This table shows the location wise categorization of } \\
\text { the abscesses and the number of patients in each category. }\end{array}$ \\
\hline
\end{tabular}

\begin{tabular}{|l|c|c|c|}
\hline Intrahepatic & 32 & 32 & $100 \%$ \\
\hline Iliopsoas & 24 & 16 & $66.6 \%$ \\
\hline Splenic & 2 & 2 & $100 \%$ \\
\hline Renal & 4 & 4 & $100 \%$ \\
\hline Subdiaphragmmatic & 26 & 22 & $84.6 \%$ \\
\hline Paracolic gutter & 6 & 4 & $66.6 \%$ \\
\hline Sub hepatic & 8 & 8 & $100 \%$ \\
\hline
\end{tabular}

Table-2: The table shows the different locations of abscesses which were subjected to drainage in this study, along with the percentage of patients in whom the drainage proved entirely successful

\begin{tabular}{|l|c|l|}
\hline Location of abscess & $\begin{array}{c}\text { Number } \\
\text { of } \\
\text { failures }\end{array}$ & Reasom for failure \\
\hline Intrahepatic & 2 & $\begin{array}{l}\text { Early abscess which were } \\
\text { phlegmonous }\end{array}$ \\
\hline Psoas & 4 & $\begin{array}{l}\text { Viscous contents, did not } \\
\text { drain completely }\end{array}$ \\
\hline Subdiaphragmmatic & 4 & $\begin{array}{l}\text { Multiloculated with vis- } \\
\text { cous contents }\end{array}$ \\
\hline Paracolic gutter & 2 & Fistula with the bowel \\
\hline $\begin{array}{l}\text { Table-3: This illustrates the location of the 12 failures in the } \\
\text { abscess drainage along with the probable reason for failure }\end{array}$ \\
\hline
\end{tabular}

months for any recurrence.

\section{STATISTICAL ANALYSIS}

Descriptive statistics like mean and percentages were used to interpret the data.

\section{RESULTS}

The number of abscesses and the location was categorization of the abscesses is illustrated in Table 1. The predominant location of the abscesses were intrahepatic, iliopsoas (cold abscesses) and subdiaphragmmatic. Category wise successful drainage percentage of the different abscesses in different location is shown in Table 2.

Amongst the 102 drainages, 70 were done under $\mathrm{CT}$ guidance and 32 under US guidance. The procedure was considered successful if there was complete drainage of the abscess with significant clinical improvement, and if the patient had no necessity of intraoperative drainage thereafter. In this study, there was a success percentage of $84.3 \%$ (86 out of 102).

Amongst the 102 drainages performed, 12 were not successful resulting in a failure rate of (11.7\%) as per Table 3. Amongst the 102 drainages, 8 abscesses recurred (Table 4).

So the recurrence rate in this study was $7.8 \%$. Precipitate withdrawal of drainage catheter caused two subdiaphragmmatic collections to recur, redrainage was done and was successful. Amongst the 102 drainages, complications developed in 12 patients and so the complication rate of (11.8\%).

\section{DISCUSSION}

Image guided percutaneous drainage have been established as a standard and safe technique for therapeutically draining intra-abdominal abscesses.

With this study, the aim was to establish more precisely the modality of image guidance, the selection criteria and the factors which contribute to higher success rate.

With this study a success rate of $84.3 \%$ has been demonstrated. The average success rate has been $70 \%$ in prior studies.

All 32 intrahepatic abscess, 16 of 24 iliopsoas collections, all 4 renal abscesses, 22 of 26 subdiaphragmmatic abscess, all two splenic abscess, four of six paracolic gutter abscess and all the subhepatic abscesses were drained successfully. ${ }^{21}$

Intrahepatic and intrasplenic abscesses had a 100\% success rate, as did subhepatic abscesses. The interesting fact about this was the subhepatic abscesses were a result of ruptured liver abscess which then had contained in the subhepatic region.

Another fact to note was, the guiding modality in all successful drainages were a combination of ultrasound and CT. Ultrasound along with color doppler was used for initial needle and catheter insertion. The further advancement of the catheter and the guidewire to access the posterior dependant

\begin{tabular}{|l|c|l|l|}
\hline Location & Number of patients & Reason & Treated by \\
\hline Iliopsoas & 4 & Non healing of the primary source in the vertebrae & Redrained \\
\hline Subdiaphragmmatic & 2 & Precipitate withdrawal of drainage catheter & Redrained \\
\hline Paracolic gutter & 2 & Fistula & Surgery \\
\hline \multicolumn{2}{|c|}{ Table-4: This illustrates the location of the 8 recurrent abscesses, probable reason for recurrence and the management } \\
\hline
\end{tabular}


wall of the abscess was facilitated by CT acquisitions. Post procedural check was done with a comprehensive CT screening.

We had a failure rate of $11.7 \%$ in our study. One liver absces had phlegmonous areas with high viscous contents. A $10 \mathrm{~F}$ catheter did not drain successfully. Catheter was upsized to $12 \mathrm{~F}$, but complete drainage was still not achieved, and the patient was taken up for open surgical drainage. This failure rate of $11.7 \%$ compares very favorably with the failure rates ranging from $12-27 \%$ in the literature. ${ }^{22,23}$ Fistulous bowel communication happened in two paracolic gutter abscesses, and the abscesses recurred. The patients were then surgically drained. ${ }^{24}$

Our recurrence rate in this study was $7.8 \%$. Precipitate withdrawal of drainage catheter caused two subdiaphragmmatic collections to recur, redrainage was done and was successful. Iliopsoas abscesses in our study were secondary to infective lumbar spondylodiscitis. Therefore, even though the abscesses were completely drained during the procedure, since the primary source had not completely healed, the abscesses recurred.

Our complication rate was 11.8\% (12 patients). In 6 of these 12 patients, the complication was sepsis. Broad spectrum, intravenous antibiotics were a mandatory part of our protocol, and they were started before the abscess wall was traversed, so as to prevent seeding of the infection into adjacent organs or the peritoneum. In spite of this precaution, 6 of our patients developed bacteremia, which was then controlled with medications. Infective strains not sensitive to the particular antibiotics were presumed to be the cause of this complication. High placed liver abscesses and splenic abcesses had to be accessed in an intercostal approach, which resulted in injury or infection to the pleural cavity. These patients were successfully treated with intercostals pleural drainage.

Most of our study patients were either post surgical or significantly ill, which deemed them relatively unfit for general anesthesia and consequently unfit for open surgical drainage. A few of our study patients were healthy, but percutaneous drainage was preferred over surgical drainage due to reduced morbidities. ${ }^{25,26}$ The advantages of image guided percutaneous drainage over open surgical drainage are

a No risk of contamination or spillage intraperitoneally.

b No need for general anesthesia.

c Increased patient compliance.

d Fewer complications.

e Similar success percentage

f Easier post procedural care

\section{CONCLUSION}

With this study, we concluded that image guided intraabdominal abscess catheter drainage is the definitive management option for abscesses in intrahepatic, intrasplenic, renal and subhepatic locations. Percutaneous drainage is the preferred management option in iliopsoas abscesses and subdiaphragmmatic abscesses. However, paracolic abscesses with the risk of fistulous enteric communication are better treated surgically.
A combined imaging guidance of ultrasound, CT and occasionally fluoroscopy provided a precise planning route, easier access, better abscess cavity entry, better access to dependant abscess wall, prevented adjacent organ damage, reduced internal hemorrhage, picked up complications early and ensured catheter position post procedurally.

Careful patient selection, meticulous preprocedural planning, judicious use of guiding imaging modalities, strict procedural asepsis, skilled technique and stringent post procedural aftercare can cumulatively contribute to even better success rates in future.

\section{REFERENCES}

1. Altemeier, et al. Intraabdominal abscess. Am J Surg. 1973; 125(2): 70-79.

2. Mel, et al. Classification, diagnosis and treatment of subphrenic abscess. Rev Surg., 1971; 28 (4): 1-21.

3. Bydder, et al. Computed tomography in diagnosis of abdominal abscess. CT, 1980; 4 (6): 132-145.

4. Casola, et al. Abscesses in Crohn disease: percutaneous drainage. Radiology, 1987; 163 (1):19-22.

5. Connel, et al. Upper abdominal abscess: a continuing and deadly problem. AJR, 1980; 134 (5):759-765.

6. Crass, et al. Bedside drainage of abscesses with sonographic guidance in the desperately ill. AIR, 1982;139 (4):183-185.

7. Deveney, et al. Improved treatment of intra-abdominal abscess: a result of improved localization, drainage and patient care, not technique. Arch Surg., 1988; 123 (6):1126-1 129 .

8. Dixon GD. Combined CT and fluoroscopic guidance for liver abscess drainage. AJR, 1980;135 (2):397-99.

9. Doust, et al. Ultrasonic distinction of abscesses from other intra-abdominal fluid collections. Radiology, 1977; 125 (1): 213-218.

10. Fry, et al. Reoperation for intra- abdominal abscesses. Surg Clin North Am. 1991; 71 (5): 159-174.

11. Gazelle, et al. Pelvic abscesses: CT guided transrectal drainage. Radiology, 1991; 181(6): 4951 - 4052.

12. Gerzof, et al. Percutaneous catheter of abdominal abscess guided by ultrasound and computed tomography. AIR, 1979; 133(4): 18 - 19.

13. Gerzof, et al. Percutaneous abscess drainage. Semin Roentgenol., 1981; 16(2): 62-71.

14. Gerzoff, et al. Percutaneous catheter drainage of abdominal abscesses: a five year experience. NEJM, 1981; 305(1): 653-657.

15. Gronvall, et al. Ultrasound-guided drainage of fluidcontaining masses using angiographic catheterization techniques. AJR, 1977; 129(4): 9974-002.

16. Haaga, et al. CT-guided percutaneous aspiration and drainage of abscess. AJR, 1980; 135(5): 1187-1194.

17. Halasz, et al. Drainage of intra- abdominal abscesses: tactics and choices. Am J Surg., 1983; 146(1): 112-114.

18. Hemming A, Davis NL, Robbins RE. Surgical versus percutaneous drainage of intra-abdominal abscesses. Am J Surg., 1991; 161(2): 593-595.

19. Jaques, et al. CT features of intra- abdominal abscesses:prediction of successful percutaneous drainage AJR, 1986; 146(4): 1041-1045.

20. Jeffrey, et al. Percutaneous drainage of periappendiceal 
abscesses: review of 20 patients. MR, 1987; 149(1): 5962.

21. Johnson, et al. Treatment of abdominal abscesses. Ann Surg., 1981; 194(2): 510-519.

22. Knochel, et al. Diagnosis of abdominal abscess with computed tomography, ultrasound and 111 in leucocyte scans. Radio1ogy, 1980; 137(1): 425-432.

23. Lamêris, et al. Ultrasound-guided percutaneous drainage of intra- abdominal abscesses. Br J Surg., 1987; 74(5): 620-623.

24. Malangoni, et al. Factors influencing the treatment of intra-abdominal abscesses. Am J Surg., 1990; 159(1): 167- 171.

25. Anita S, Senthil Anbumani. Study of technique, advantages and effectiveness of percutaneous drainage of intra-abdominal abscesses. IAIM, 2017; 4(3): 30-36.

26. Martin, et al. Percutaneous drainage of postoperative intra-abdominal abscess. AJR, 1982; 138(4): 13-15.

Source of Support: Nil; Conflict of Interest: None

Submitted: 20-08-2018; Accepted: 01-09-2018; Published online: 09-09-2018 